\title{
Mixed Integer Linear Programming for Maintenance Scheduling in Power System Planning
}

\author{
S.M. Hussin ${ }^{1}$, M.Y. Hassan ${ }^{2}$, L. Wu ${ }^{3}$, M.P. Abdullah ${ }^{4}$, N. Rosmin ${ }^{5}$, M.A. Ahmad ${ }^{6}$ \\ ${ }^{1,2,4,5}$ POWER Department, Faculty of Electrical Engineering, Centre of Electrical Energy System (CEES), \\ Universiti Teknologi Malaysia (UTM), 81310 Johor Bharu, Johor \\ ${ }^{3}$ Department of Electrical \& Computer Engineering, Clarkson University, Potsdam, NY 13699-5720, USA \\ ${ }^{6}$ System Operations Department, Transmission Division, Tenaga Nasional Berhad, Kuala Lumpur, Malaysia
}

\begin{tabular}{l}
\hline \hline Article Info \\
\hline Article history: \\
Received Mar 1, 2018 \\
Revised Apr 27, 2018 \\
Accepted May 1, 2018 \\
\hline
\end{tabular}

\section{Keywords:}

MILP

LR

Generation maintenance scheduling

Transmission maintenance scheduling

Security-constrained unit commitment

\begin{abstract}
This paper discussed the merit of mixed-integer linear programming (MILP)based approach against Lagrangian relaxation (LR)-based approach in solving generation and transmission maintenance scheduling problem. MILP provides a straightforward solution by formulating coupling constraints equations so that these sub-problems can be solved simultaneously without involving multipliers. In LR-based approach, generation and transmission maintenance scheduling, and security-constrained unit commitment have been solved individually and the integration was realized through a series of multipliers which has caused computational burden to the system. Numerical case studies were evaluated on the 6-bus system. A comparative study is carried out between the MILP and LR approaches. Simulation results indicate that the maintenance schedule derived by the proposed MILP approach outperforms the LR in terms of operational cost savings and gap tolerance. The operating cost could be saved up to $5 \%$ and the gap tolerance achieved is $0.01 \%$ as compared to $0.14 \%$ by LR.
\end{abstract}

Copyright (C) 2018 Institute of Advanced Engineering and Science. All rights reserved.

Corresponding Author:

S.M.Hussin,

POWER Department, Faculty of Electrical Engineering,

Centre of Electrical Energy System (CEES), Universiti Teknologi Malaysia (UTM),

81310 Johor Bharu, Johor.

Email: maherah@fke.utm.my

\section{INTRODUCTION}

In general, preventive maintenance is a periodic inspection procedure carried out on equipment components, in order to reduce the possibility of failure. It is done on the existing online equipment which need to be temporarily shut down for maintenance work. Schedules of maintenance tasks need to be managed efficiently by the system operator so that the equipment is maintained at the most appropriate time periods. Maintenance of generation or transmission line can cause changes in the output of generation units, which in turn will affect the cost of MW production. For example, maintenance of units can trigger the use of more expensive and/or inefficient generation plants to supply the demand. Another fact is that taking equipment out of service for maintenance is always associated with a possible increase in the risk of overall system operation during the maintenance period. In this sense, the system operator should oversee the overall operation of the system before performing maintenance so that any maintenance schedule will not cause disruption in the system or significant increase operating costs [1].

The most viable method to optimize maintenance scheduling is mathematical programming technique such as dynamic programming (DP) [2], Benders decomposition (BD) [3-5], integer programming (IP) [6-7], Lagrangian relaxation (LR) [8-11], and mixed integer linear programming (MILP) [12-20]. Among these techniques, most of the coordinated generation and transmission maintenance scheduling problem has been solved by LR-based approach. The LR technique solves maintenance scheduling problems 
by relaxing the explicit coupling constraints. In this technique, each coupling constraint is in respect of one multiplier; hence the number of multiplier involved will be increased as coupling constraints increase [9]. The efficiency of the solution process highly depends on the value of the multiplier, thus the selection of an appropriate method for updating the multiplier is crucial. Besides, a good technical background is necessary to determine the trust region of the multiplier; otherwise the procedure would take a longer computational time to converge. Moreover, LR approach usually tackles the problem on the day-by-day basis, hence ramp rate constraints of generators on consecutive days have been relaxed, which would jeopardize the loading and unloading characteristics of a generator.

In view of these shortcomings, the MILP-based approach is implemented to solve the coordinated maintenance scheduling problem. The key benefits of the proposed approach are simplicity and guaranteed optimal solution. In addition, it could accurately address ramp rate constraints of generators on consecutive days. It is designed with appropriate level of granularity by considering all operation constraints, both unitwise and system-wise, such that it is comparable to a real practical system. The co-optimization of generation maintenance scheduling, transmission maintenance scheduling, and unit commitment sub-problems are solved simultaneously by a one-shot solution. Unlike LR approach, where the sub-problems are optimized individually before integrated by using multipliers.

The remaining of the paper is organized as follows. Section 2 highlights MILP and LR -based solution approaches. Numerical case studies using 6-bus system is discussed in Section 3, and the conclusions are drawn in Section 4.

\section{PROPOSED MILP AND CURRENT LR SOLUTION PROCEDURES}

LR-based approach is used as a benchmark in this study as it has been used widely in solving coordinated generation and transmission maintenance scheduling problem. Figure 1 shows the solution procedure of the LR-based approach. In this procedure, GMS, TMS, and SCUC sub-problems are initially solved independently. Then, the coordination of these sub-problems is realized through a series of multipliers. Based on the flowchart, $\mu$ is a multiplier for the coupling constraint of generation maintenance $X$, and unit commitment, $I$, meanwhile, $\pi$ is a multiplier for the coupling constraint of the transmission maintenance schedule, $L$ and transmission line state, $M$. In this solution process, multipliers are updated until the final solution is reached. The sub-gradient method and bundle method are the commonly used approaches for updating multipliers [8 -10]. Usually, initial values of multipliers need to be carefully chosen in order to determine the trust region of the multipliers. Otherwise, the procedure could take long computational time to converge.

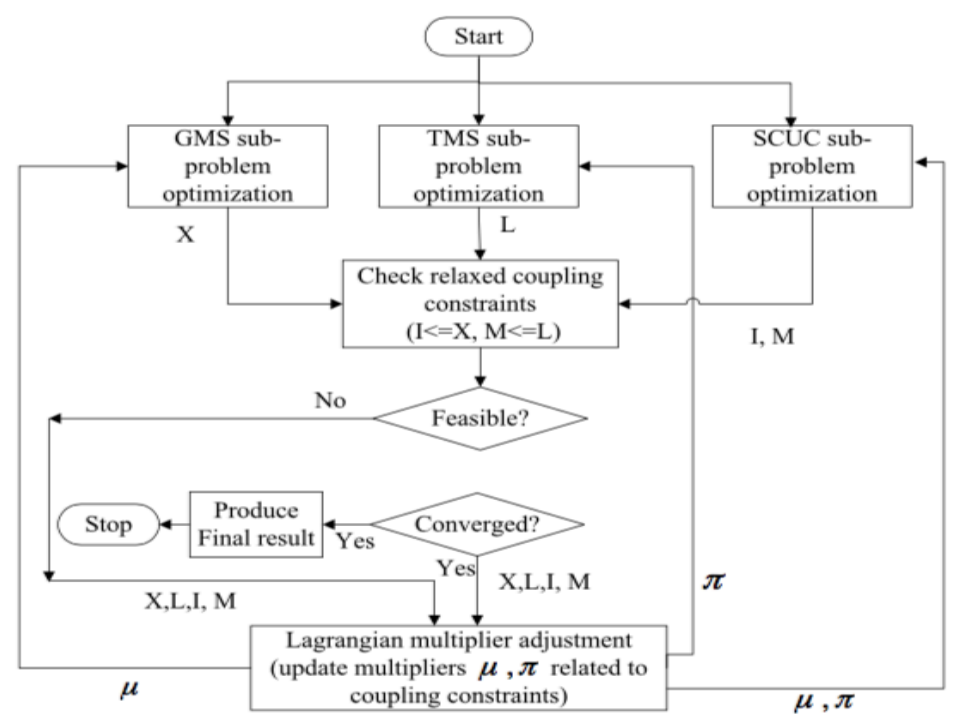

Figure 1. Flowchart of the LR procedure

However, in the proposed MILP-based approach, all these sub-problems are solved simultaneously in which the coordination is realized through coupling constraints. This approach does not involve any 
multiplier where its coupling constraints are directly embedded in the formulation. Since the proposed MILP has involved a large number of variables and constraints, the solution is achieved with the aid of commercial solver, CPLEX. The general procedure of the proposed MILP-based approach is illustrated in Figure 2. To reduce dimensionality problem especially in solving large scale system, line limit constraints are added only if they have been violated. Table 1 summarized the differences between the proposed MILP based approach and the previous LR based approach.

Table 1. Comparison between the MILP and the LR based approach

\begin{tabular}{|c|c|}
\hline LR-based approach (Figure 1) & Proposed MILP-based approach (Figure 2) \\
\hline All the sub-problems are solved individually. & All the sub-problems are solved in a simultaneous way. \\
\hline $\begin{array}{l}\text { Multipliers are involved to relax coupling constraints in which } \\
\text { they exist between sub-problems. An additional method is } \\
\text { required for updating multipliers. }\end{array}$ & No multipliers are involved for solving coupling constraints. \\
\hline $\begin{array}{c}\text { Solves the problem on a day-to-day basis with hourly time } \\
\text { resolution. }\end{array}$ & $\begin{array}{c}\text { Solves the problem of the duration of pre-specified maintenance } \\
\text { planning with hourly time resolution. }\end{array}$ \\
\hline
\end{tabular}

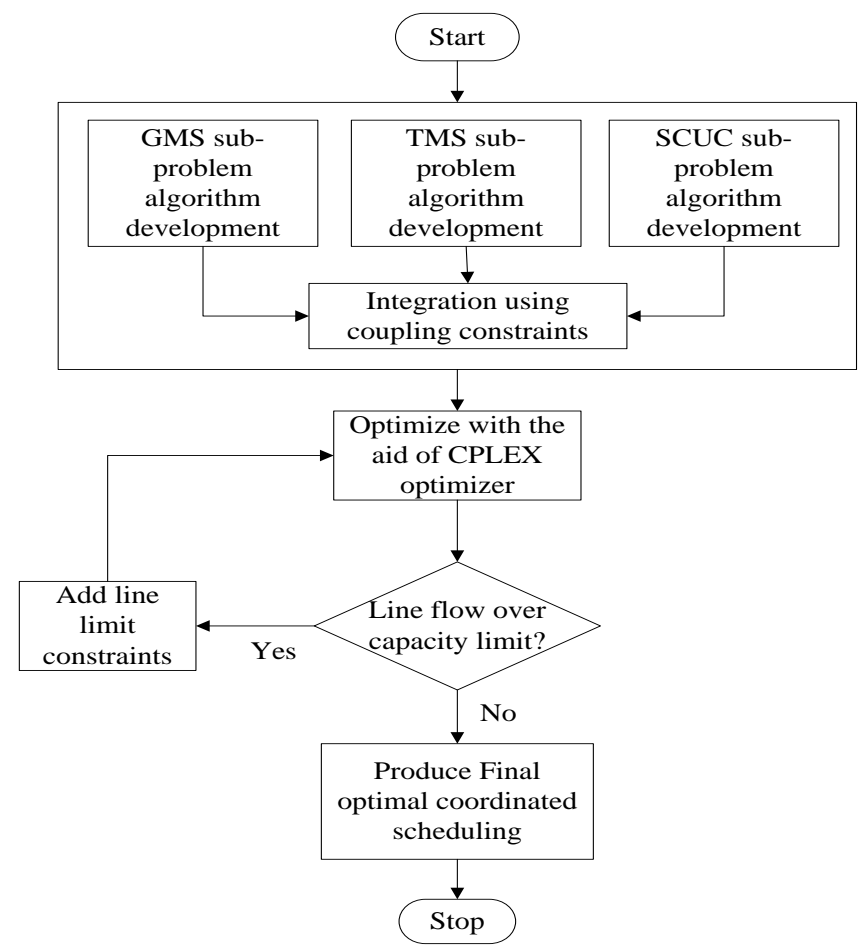

Figure 2. Flowchart of the proposed MILP solution procedure

\section{CASE STUDIES}

The performance of the proposed model is evaluated through several cases on a 6-bus system. In this study, the proposed MILP-based schedule approach is compared to the schedule suggested in [9], where the LR-based approach is implemented. Due to crew constraints, generation units and transmission lines may not be scheduled for maintenance simultaneously.

The 6-bus system consists of three generators, seven transmission lines, and three load demands as presented in Figure 3. Chronological load profile over a one-week planning horizon is depicted in Figure 4, in which the peak load is $270 \mathrm{MW}$. Tables 2-3 show the generator information and maintenance data, respectively. Four cases are discussed in this study:

Case 0: Unit commitment without equipment maintenance (base case)

Case 1: Unit commitment with generator maintenance.

Case 2: Unit commitment with transmission maintenance.

Case 3: Unit commitment with co-optimized generator and transmission maintenance. 


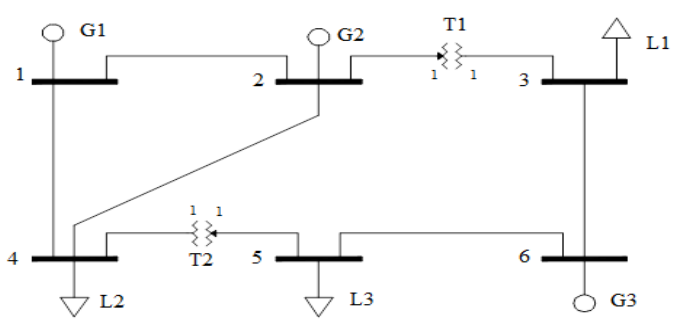

Figure 3. The 6-bus system

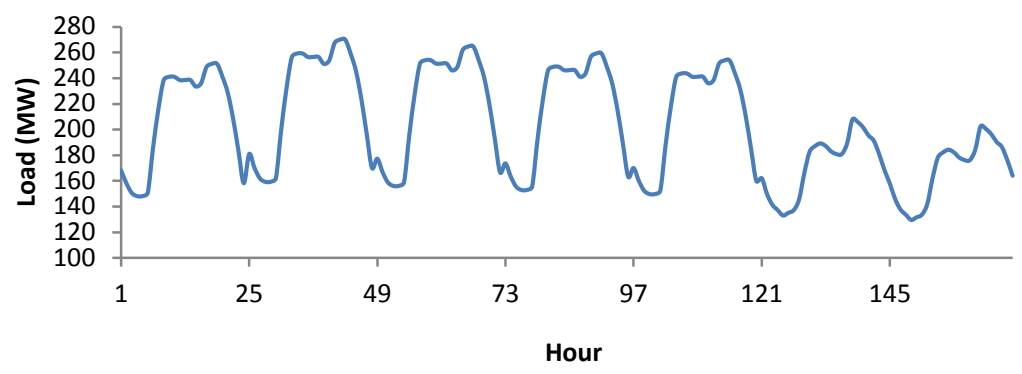

Figure 4. Load profile over 168 hours of the planning horizon

Table 2. Generator cost and operating data

\begin{tabular}{|c|c|c|c|c|c|c|c|c|c|}
\hline Unit & a (MBtu/h) & $\begin{array}{c}\mathrm{b} \\
(\mathrm{MBtu} / \mathrm{MWh})\end{array}$ & c $\left(\mathrm{MBtu} / \mathrm{MW}^{2} \mathrm{~h}\right)$ & $\begin{array}{l}\text { Pmin } \\
\text { (MW) }\end{array}$ & $\begin{array}{l}\text { Pmax } \\
(\mathrm{MW})\end{array}$ & $\begin{array}{c}\text { Ramp } \\
\text { Rate } \\
(\mathrm{MW} / \mathrm{h})\end{array}$ & $\begin{array}{l}\text { Min-up } \\
\text { Time (h) }\end{array}$ & $\begin{array}{c}\text { Min- } \\
\text { down } \\
\text { Time (h) }\end{array}$ & $\begin{array}{c}\text { Initial } \\
\text { State (h) }\end{array}$ \\
\hline G1 & 176.9 & 13.5 & 0.00045 & 100 & 220 & 55 & 4 & 4 & 4 \\
\hline G2 & 129.9 & 32.6 & 0.001 & 50 & 150 & 50 & 2 & 3 & 2 \\
\hline G3 & 137.4 & 17.6 & 0.005 & 20 & 100 & 40 & 1 & 1 & 1 \\
\hline
\end{tabular}

Table 3. Equipment maintenance data

\begin{tabular}{ccc}
\hline Equipment & Maintenance Window & Outage Duration (h) \\
\hline G1 & Mon-Sun & 24 \\
G2 & Mon-Sun & 24 \\
G3 & Mon-Sun & 24 \\
L1-2 & Tue - Sat & 24 \\
\hline
\end{tabular}

Thest are the findings summarized for all cases. For further analysis, the results have been compared to the LR-based approach as it was obtained in [8].

Case 0). In this base case, unit commitment is solved without considering any equipment maintenance. Hourly unit commitment results from the proposed model are compared to the schedules suggested by the LR-based approach as illustrated in Figure 5. The schedules show that both models commit the cheapest unit G1 while allowing the most expensive unit G2 to shut down over the entire week. Due to the capacity limit of G1, G3 is also triggered to operate at the time unit G1 could not support the load on its own. The slight difference in the committed hours of unit G3 would result in a notable production cost difference. That is, MILP yields a lower operating cost compared to LR, which are \$510,543.18 and $\$ 520,732$, respectively.

Case 1). Unit commitment and generator maintenance schedules suggested by the proposed MILP and LR-based approach for this case are described in Figure 6. It shows that both approaches schedule G1 for maintenance on Sunday (i.e. Hours 145-168), which is during the lowest demand period. Meanwhile, the proposed model scheduled G3 during hours 118-141, while is hours 121-144 in LR-based approach. Maintenance scheduling of G2 can be done at any time throughout the week, as it is not operated over the entire week and will not impact the systems operation cost. The proposed model schedules G2 during hours 2-25, while LR suggested during hours 97-120. The schedules suggested by the proposed MILP yield a lower operation cost of $\$ 561,119.95$, compared to $\$ 566,538$ of the LR-based approach. The cost-saving achieved in this case is about $\$ 5,418.05$. 


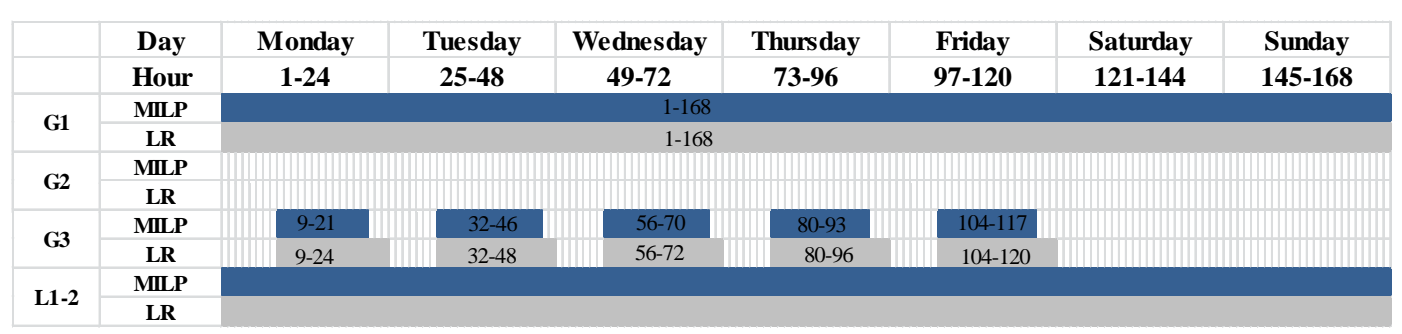

Figure 5. Unit commitment results from the MILP and LR approaches in Case 0

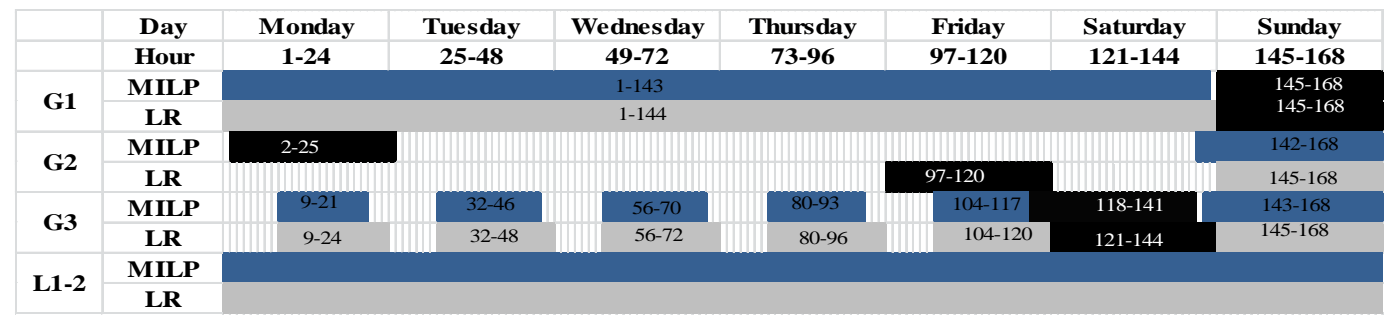

Figure 6. Unit commitment and generator maintenance scheduling for the MILP and LR approaches in Case 1

From the investigation, it is found that the LR-based approach does not satisfy ramp rate constraints of generators on consecutive days as it tackles the problem on a day-by-day basis, and such constraints have been relaxed. According to the LR schedule (refer to Figure 6), one hour before G1 started undergoing maintenance (i.e. hour 144), only G1 has supplied the load, where the load at that time is 168.39 MW. It shows that G1 has been switched off instantly which does not satisfy the ramp rate constraints. The constraints stated that the unloading of a unit must be done gradually within its rates and should generate at its minimum power before being totally switched off. On the other hand, the schedules suggested by the proposed MILP show that during that hour (i.e. hour 144), the load has been supplied by G2 (108.399 MW) and G3 (60 MW) as illustrated in Figure 7, hence, it shows that the dispatch of G1 has gradually decreased prior to it beginning to undergo maintenance. This is one of the reasons why maintenance of G3 has been scheduled earlier than the schedule suggested by the LR model.

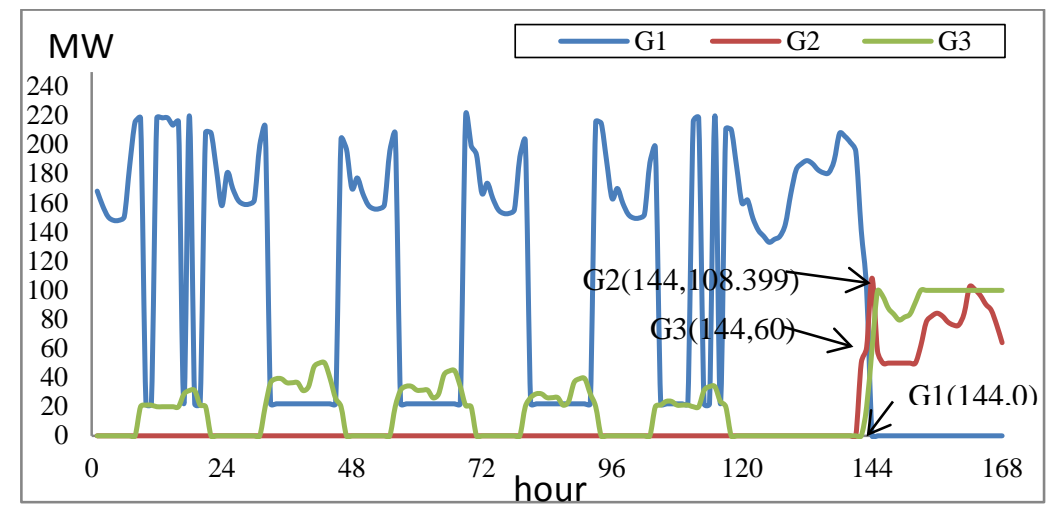

Figure 7. Economic dispatch of the proposed MILP-based approach for Case 1

Case 2). The line maintenance schedules obtained in this case for both approaches are summarized in Figure 8. In the LR-based model, line L1-2 is suggested to be maintained during hours 121-144, which would require the expensive G3 to commit during peak hours 138-140 on Saturday. The operating cost obtained by the LR approach is $\$ 522,273$. In contrast, the overall cost could be saved up to $\$ 11,195.82$ if it follows the schedule suggested by the proposed MILP model, where line L1-2 is scheduled during hours 114- 
137. This schedule will not commit G3 during these peak hours on Saturday. The operating cost obtained using the MILP-based approach is \$511,077.18.

\begin{tabular}{|c|c|c|c|c|c|c|c|c|}
\hline & Day & Monday & Tuesday & Wednesday & Thursday & Friday & Saturday & Sunday \\
\hline & Hour & $1-24$ & $25-48$ & $49-72$ & 73-96 & $97-120$ & $121-144$ & $145-168$ \\
\hline \multirow{2}{*}{ G1 } & MILP & \multicolumn{7}{|c|}{$1-168$} \\
\hline & LR & \multicolumn{7}{|c|}{$1-168$} \\
\hline \multirow{2}{*}{ G2 } & MILP & & & & & & & \\
\hline & LR & & & & & & & \\
\hline \multirow{2}{*}{ G3 } & MILP & $9-21$ & $32-46$ & $56-70$ & $80-93$ & $104-118$ & \multirow{2}{*}{\multicolumn{2}{|c|}{$138-140$}} \\
\hline & LR & $9-24$ & $32-48$ & $56-72$ & $80-96$ & $104-120$ & & \\
\hline \multirow{2}{*}{ L1-2 } & MILP & & & & & & \multicolumn{2}{|c|}{$14-137$} \\
\hline & LR & & & & & & \multicolumn{2}{|l|}{$121-144$} \\
\hline
\end{tabular}

Figure 8. Unit commitment and line maintenance schedules for the MILP and LR approaches in Case 2

Case 3). In this case, the schedules of unit commitment, generation maintenance, and transmission maintenance are optimized simultaneously. Figure 9 shows the maintenance schedule of both approaches. The result shows that there is a small difference in the starting hour for maintenance of G1 and G3 as compared to the LR-based approach. G1 is maintained one hour earlier than suggested by LR, meanwhile G3 starts two hours earlier, which is hour 119 instead of 121. For line maintenance, L1-2 is suggested during hours 114-137 instead of 97-120. The operating cost obtained in this case is $\$ 561,653.95$, which is lower than $\$ 567,607$ of the LR-based approach. That is, the cost saving achieved in this case is about \$5,953.05.

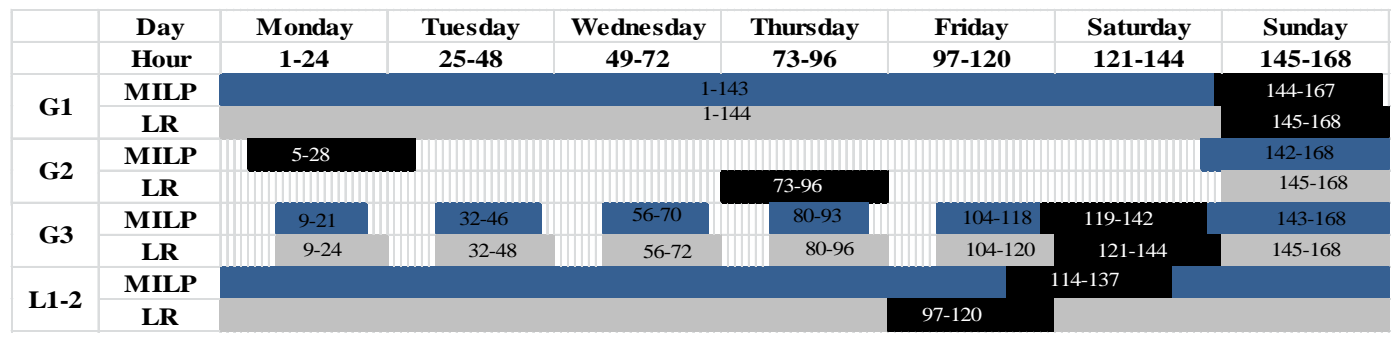

Figure 9. Unit commitment schedule and maintenance schedule for the MILP and LR approaches in Case 3

From the discussion above, it can be concluded that the schedules suggested by the proposed MILP have a better performance as compared to the LR-based approach, because it could reduce the overall operating cost. The cost saving achieved for all cases are summarized in Table 4.

Table 4. Summary of costs for all cases for the MILP and LR approaches (\$)

\begin{tabular}{llll}
\hline Case & MILP & LR & Cost Saving \\
\hline 0 & $510,543.18$ & 520,732 & $10,188.82$ \\
1 & $561,119.95$ & 566,538 & $5,418.05$ \\
2 & $511,077.18$ & 522,273 & $11,195.82$ \\
3 & $561,653.95$ & 567,607 & $5,953.05$ \\
\hline
\end{tabular}

\section{CONCLUSION}

This paper highlights the effectiveness of the proposed MILP-based approach over the LR-based approach for solving co-optimized generation and transmission maintenance scheduling with SCUC. The simulation result has shown that the overall operating cost could be saved up to $5 \%$ using the proposed model and the tolerance can be improved from $0.14 \%$ for LR based approach to $0.01 \%$. The results indicate the superiority of the MILP-based approach in solving high-dimensional maintenance problem as it can derive better solution as compared to the LR-based approach. 


\section{ACKNOWLEDGEMENTS}

The authors would like to thank Research Management Centre (RMC), Centre of Electrical Energy System (CEES), POWER department, Faculty of Electrical Engineering, Universiti Teknologi Malaysia, and the Ministry of Higher Education of Malaysia for the financial support provided under Research University Grant (RUG Tier 2) vot 14J56 to carry out this research.

\section{References}

[1] Hong, S.S., Yong, Q.K., Juan, D.L. Control Strategy Analysis on Preventive Maintenance. Indonesian Journal of Electrical Engineering and Computer Science. 2014; 12(9): 6711-6724.

[2] Prashant, K.P., Padma L.P. Dynamic Virtual Programming Optimizing the Risk on Operating System. Indonesian Journal of Electrical Engineering and Computer Science. 2014; 12(8): 6369-6379.

[3] Yellen, J., Al-Khamis, T. M., Vemuri, S. and Lemonidis, L. A Decomposition Approach to Unit Maintenance Scheduling. IEEE Transactions on Power Systems. 1992; 7(2): 726-733.

[4] Marwali, M. K. C. and Shahidehpour, S. M. A Probabilistic Approach to Generation Maintenance Scheduler with Network Constraints. International Journal of Electrical Power \& Energy Systems. 1999; 21(8): 533-545.

[5] Marwali, M. K. C. and Shahidehpour, S. M. Integrated Generation and Transmission Maintenance Scheduling with Network Constraints. IEEE Transactions on Power Systems. 1998; 13(3):1063-1068.

[6] Leou, R.C. A Flexible Unit Maintenance Scheduling Considering Uncertainties. IEEE Transactions on Power Systems, 2001; 16 (3); 552-559.

[7] S Chanda, A De. Congestion relief of contingent power network with evolutionary optimization algorithm. TELKOMNIKA (Telecommunication, Computing, Electronics and Control). 2012; 10(1): 1-8.

[8] Fu, Y., Shahidehpour, M. and Li, Z. Security-Constrained Optimal Coordination of Generation and Transmission Maintenance Outage Scheduling. IEEE Transactions on Power Systems 2007; 22(3):1302-1313.

[9] Fu, Y., Li, Z. and Shahidehpour, M. Coordination of Midterm Outage Scheduling with ShortTerm SecurityConstrained Unit Commitment. IEEE Transactions on Power Systems, 2009; 24(4):1818-1830.

[10] Wu, L., Shahidehpour, M. and Fu, Y. Security-Constrained Generation and Transmission Outage Scheduling with Uncertainties. IEEE Transactions on Power Systems. 2010; 25(3); 1674-1685.

[11] Subramani R., Vijayalakshmi C. Design and Analysis of Lagrangian Algorithm for Power Flow System using Renewable Energy Resources. Indonesian Journal of Electrical Engineering and Computer Science. 2017; 7(2); 348-355.

[12] Conejo, A. J., Bertrand, R. G. and Salazar, M. D. Generation Maintenance Scheduling in Restructured Power Systems. IEEE Transactions on Power Systems. 2005; 20(2); 984-992.

[13] Barot, H. and Bhattacharya, K. Security Coordinated Maintenance Scheduling in Deregulation Based on Genco Contribution to Unserved Energy. IEEE Transactions on Power Systems. 2008; 23(4): 1871-1882.

[14] Kovacs, A., Erdos, G., Viharos, Z.J., Monostori, L. A system for the detailed scheduling of wind farm maintenance. CIRP Annals Manufacturing Technology. 2011; 60(1): 497-501.

[15] Pandzic, H., Conejo, A. J., Kuzle, I. and Caro, E. Yearly Maintenance Scheduling of Transmission Lines Within a Market Environment. IEEE Transactions on Power Systems, 2012; 27(1): 407-415.

[16] Badri, A. and Niazi, A. N. Preventive Generation Maintenance Scheduling Considering System Reliability and Energy Purchase in Restructured Power System. Journal of Basic and Applied Scientific Research. 2012; 2(12): 12773-12786.

[17] Canto, S. P. and Romero, J. C. R. A Model for the Preventive Maintenance Scheduling of Power Plants including Wind Farms. Reliability Engineering \& System Safety. 2013; 119: 67-75.

[18] Jost, V., Savourey, D. An 0-1 Integer Linear Programming Approach to Schedule Outages of Nuclear Power Plants. Electricite De France, 2013; 16(6): 551-566.

[19] Pandzic, H., Conejo, A. and Kuzle, I. An EPEC Approach to the Yearly Maintenance Scheduling of Generating Units. IEEE Transactions on Power Systems. 2013; 28 (2): 922-930.

[20] Mollahassani-pour, M., Abdollahi, A. and Raashidinejad, M. Application of a Novel Cost Reduction Index to Preventive Maintenance Scheduling. Electrical Power and Energy Systems. 2014; 56: 235-240. 